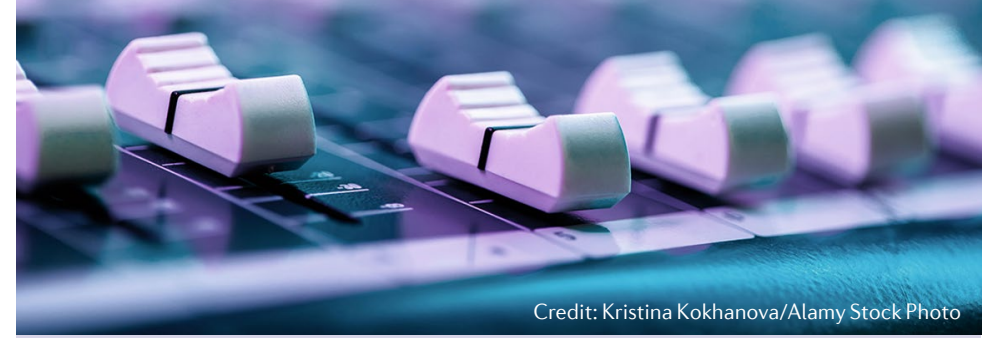

OSTEOARTHRITIS

\section{TET1: an epigenetic controller of OA}

Osteoarthritis (OA) is a heterogeneous disease mediated by multiple molecular pathways and governed by a complex interplay between various genetic, epigenetic and environmental factors. New findings published in Science Translational Medicine implicate the epigenetic regulator ten-eleven translocation 1 (TET1) as an important activator of multiple OA-associated pathways and as an attractive therapeutic target.

TET enzymes catalyse the initial step of DNA demethylation by converting 5-methylcytosine into 5-hydroxymethylcytosine ( $5 \mathrm{hmC})$, an epigenetic process associated with gene activation. Previous evidence had shown that $5 \mathrm{hmC}$ accumulates on OA-related genes in osteoarthritic chondrocytes. To investigate this process further, the authors of the new study mapped changes in the $5 \mathrm{hmC}$ epigenome in mice following induction of OA by destabilization of the medial meniscus (DMM), with and without the expression of Tet1.

In wild-type mice, $\mathrm{OA}$ induction was accompanied by a genome-wide accumulation of $5 \mathrm{hmC}$, predominantly in gene bodies or intergenic regions, and an upregulation in expression of hundreds of genes. Notably, almost half of the upregulated genes gained sites of $5 \mathrm{hmC}$ accumulation, including genes involved in WNT signalling, protein kinase A signalling and inositol metabolism.

The majority of $5 \mathrm{hmC}$ deposition was lost in mice lacking TET1. Importantly, loss of Tet 1 impeded the initiation and development

of DMM-induced OA, including the deterioration of cartilage and osteophyte formation.

TET1 activated various pathways important in OA pathogenesis, including WNT signalling, metalloproteinases and STAT3 signalling. Indeed, shRNA-mediated knockdown of TET1 in chondrocytes from patients with OA decreased the expression of $M M P 3$ and $M M P 13$.

To provide proof of principle that modulating TET1 activity is a promising therapeutic strategy, the researchers tested a small molecular inhibitor of TET1, 2-hydroxyglutarate (2-HG). Intra-articular injection of 2-HG after DMM surgery stalled OA progression in mice, and this inhibitor could replicate the effects of TET1 knockdown in osteoarthritic chondrocytes in vitro.

Jessica McHugh

panied by a genome-wide accumulation of $5 \mathrm{hmC}$

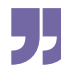

ORIGINAL ARTICLE Smeriglio, P. et al. Inhibition of TET1 prevents the development of osteoarthritis and reveals the $5 \mathrm{hmC}$ landscape that orchestrates pathogenesis. Sci. Transl Med. 12, eaax2332 (2020)

RELATED ARTICLE Rice, S. J. et al. Interplay between genetics and epigenetics in osteoarthritis. Nat. Rev. Rheum. 16, 268-281 (2020)

\section{Targeting FLS signalling in RA}

Fibroblast-like synoviocytes (FLS) can promote joint inflammation and destruction in rheumatoid arthritis (RA) through the production of pro-inflammatory mediators such as IL-15 and dickkopf-related protein 1 (DKK1). New findings published in Arthritis \& Rheumatology highlight the involvement of a signalling axis downstream of discoidin domain receptor 2 (DDR2) in this process.

Previous studies had suggested that DDR2, a receptor tyrosine kinase (RTK), is expressed in FLS and contributes to cartilage and bone destruction in RA. In the new study, the researchers found that the expression of DDR2 correlated with the expression of IL-15 and DKK1 both in FLS from patients with RA (RA FLS) and in mice with collagen antibody-induced arthritis (CAIA).

Following collagen antibody treatment, Ddr2 ${ }^{-1-}$ mice had milder arthritis than wild-type mice and reduced expression of both IL-15

and DKK1. Restoring the expression of DDR2 in the joints of Ddr2 $2^{-1-}$ mice treatment with

WRG-28 was associated with reduced clinical arthritis scores using a DDR2-expressing adenovirus increased the arthritis severity score as well as the expression of IL-15 and DKK1.

In vitro experiments in RA FLS identified the long non-coding RNA $\mathrm{H} 19$ as a downstream target of the DDR2 signalling cascade. $\mathrm{H} 19$ could then interact with and downregulate miR-103a, a microRNA previously shown to be downregulated in RA FLS. Notably, the predicted targets of this microRNA included IL15 and $D K K 1$, and, indeed, data from dual luciferase reporter assays suggested that miR-103a could directly target and repress the expression IL15 and DKK1.

"DDR2 can be blocked by several FDA-approved RTK inhibitors, such as dasatinib and imatinib, and a recent study has shown that inhibition of DDR2 by dasatinib attenuates inflammation severity and bone destruction in mice with CAIA and RA FLS," explains corresponding author Wei Zhang. Given the low specificity of dasatinib for DDR2, Zhang and colleagues explored the potential of a more recently developed small molecule inhibitor of DDR2, WRG-28.

In the CAIA model, treatment with WRG-28 was associated with reduced clinical arthritis scores, as well as reduced levels of inflammatory cell infiltration and destruction of cartilage and bone. In line with the proposed DDR2-H19-miR-103a signalling axis, WRG-28 treatment was also associated with decreased expression of $\mathrm{H} 19$, IL-15 and DKK1 and increased expression of miR-103 in the ankle joints of the mice.

\section{Jessica McHugh}

ORIGINAL ARTICLE Mu, N. et al. Blockade of discoidin domain receptor 2 as a strategy for reducing inflammation and joint destruction in rheumatoid arthritis via altered interleukin-15 and Dkk-1 signaling in fibroblast-like synoviocytes. Arthritis Rheumatol. https://doi.org/10.1002/ art.41205 (2020)

RELATED ARTICLES Nygaard, G. \& Firestein, G. S. et al. Restoring synovial homeostasis in rheumatoid arthritis by targeting fibroblast-like synoviocytes. Nat. Rev. Rheum. 16, 316-333 (2020) 